\title{
Analysis of Capnogram Using Linear Predictive Coding (LPC) to Differentiate Asthmatic Conditions
}

\section{Kazemi and MB Malarvili}

Faculty of Biomedical Engineering and Health Science, Universiti Teknologi Malaysia, Malaysia

\begin{abstract}
In this paper, an analysis of capnogram to differentiate asthmatic and non-asthmatic patients is presented by using linear predictive coding (LPC) technique. In the previous studies, manual study on capnogram signal has been conducted by several researchers. All previous researches show significant correlation between the capnogram and asthmatic patient. However all of them are just manual study conducted through the conventional time domain method. In this preliminary, a number of 8 LPC coefficients $\left(\alpha_{1}-\alpha_{8}\right)$ for both asthmatic (CAP) and non-asthmatic patients' capnogram (CNP) are extracted. Usefulness and performance of these coefficients to differentiate the asthmatic conditions by means of receiver operating characteristic (ROC) curve analysis are shown. Our preliminary results show that $\alpha_{3}, \alpha_{4}, \alpha_{5}$, and $\alpha_{6}$ can be used to distinguish the asthmatic conditions.
\end{abstract}

Keywords: Asthma; Capnography; Capnogram; LPC

\section{Introduction}

Asthma is a chronic inflammatory disease of the bronchial tubes in which the bronchi narrow excessively and generally reversibly in response to a variety of stimuli. It occurs in $3 \%-5 \%$ of all people at sometimes in their lives [1].

In many respiratory diseases, particularly in asthma, the resistance to airflow becomes great during expiration, causing tremendous difficulty in breathing. It has led to the concept called maximum expiratory flow, which can be defined as follows: when a person expires with great force, the expiratory airflow reaches a maximum flow beyond which the flow cannot be increased any more even with greatly increased additional force, and this characteristic of asthma is the principle of its diagnosis [1].

Traditionally, one of the pulmonary tests is to make a record on a spirometer of the forced expiratory vital capacity (FVC). Spirometry involves a maximal inspiration followed by a rapid, forceful, and complete exhalation until there is absolutely no more air to blow out [2]. These results generally come with two different graphic displays in which the first is the spirogram and the second is the flow-volume curve. One of the information regarding to the flow-volume curve is the point of maximal expiratory flow, the same as the peak expiratory flow rate (PEFR). It is the expiratory flow at the moment that is the fastest during the entire exhalation and the value that measured by peak flow meters [2].

Spirometry and peak flow meter are useful diagnostic tools, but there are some complications and contraindications that among these are dizziness, chest pain, coughing, bronchospasm, and oxygen desaturation due to the interruption of oxygen therapy. The reliable results cannot be obtained if a patient is unable to understand the instructions, has chest pain preventing a forceful effort, or does not choose to cooperate. Also there are more limitations that could be found in [3].

Capnography is a new method used to monitor the asthmatic condition [4]. It is able to show the different respiratory situation of patient including asthma and is taken while the patient is breathing as comfortably as able, without requiring following any breathing instructions. A normal capnogram has four phases and an end-tidal point [5], as shown in Figure 1. Each phase reflects normal process of $\mathrm{CO}_{2}$ elimination.
The flat phase I (A_B) represents early exhalation that is relatively $\mathrm{CO}_{2}$-free. As exhalation continues, alveoli containing $\mathrm{CO}_{2}$ are increasingly recruited and exhaled with non $\mathrm{CO}_{2}$-containing gases. This creates a near vertical rising phase II (B_C). Near the termination of normal exhalation is a plateau phase III (C_D). At the end of the plateau phase is $\mathrm{D}$, the point that the measured alveolar $\mathrm{CO}_{2}$ levels best approximate $\mathrm{PaCO}_{2}$. This sampled $\mathrm{CO}_{2}$ level is known as $\mathrm{PetCO}_{2}$. As inspiration occurs, a near vertical rapidly falling phase IV (D_E) is observed. When ventilation and perfusion function normally, $\mathrm{PetCO}_{2}$ should read 2-5 mmHg higher than the $\mathrm{PaCO}_{2}$ [5].

A variety of clinical causes can lead to incomplete alveolar emptying

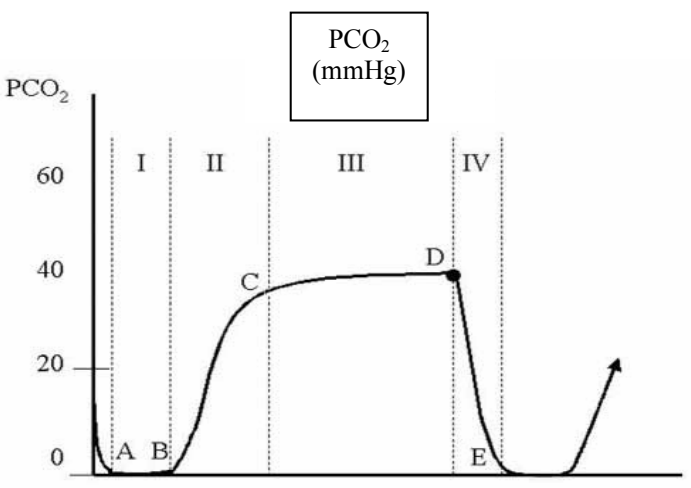

Figure 1: Normal Capnogram.

*Corresponding author: M. Kazemi, Faculty of Biomedical Engineering and Health Science, University Technology Malaysia, Malaysia, E-mail: Mohsenkazemi20@ gmail.com

Received November 21, 2011; Accepted November 30, 2011; Published December 02, 2011

Citation: Kazemi M, Malarvili MB (2011) Analysis of Capnogram Using Linear Predictive Coding (LPC) to Differentiate Asthmatic Conditions. J Tissue Sci Eng 2:111. doi:10.4172/2157-7552.1000111

Copyright: ( 2011 Yamano S, et al. This is an open-access article distributed under the terms of the Creative Commons Attribution License, which permits unrestricted use, distribution, and reproduction in any medium, provided the original author and source are credited. 
[5]. So, the true end tidal point is never reached. The capnogram of an asthmatic patient or in a patient with an obstruction in tubing or other parts of the breathing circuit appears in Figure 2(b). Notice the ascending limb of the capnogram is prolonged and is not flat, as it should be normally as shown in Figure 2(a). These changes give rise to the so called "shark's fin" morphology capnogram in patients with airway obstruction [6].

In the previous studies, manual study on capnogram signal has been conducted by several researchers. One of the first and fundamental studies in analyzing capnogram has been done by You et al. [7]. In this research, the correlations between capnographic and spirometric indices were measured in 10 healthy subjects and 30 asthmatic patients. Also the usefulness of eight descriptive indices was assessed by measuring their reproducibility and their sensitivity to airway obstruction. Three indices measured the slope of the capnogram during the various phases of expiration (S1, S2, and S3), the slope ratio $(\mathrm{SR})$ between the intermediate and the initial slopes $\left(\mathrm{S}_{2} / \mathrm{S}_{1}\right)^{*} 100$, the area ratio (AR: $\left.\left(\mathrm{A}_{1} / \mathrm{A}_{2}\right)^{*} 100\right)$ that the $\mathrm{A}_{1}$ and $\mathrm{A}_{2}$ were calculated above a threshold of $2.5 \%$ of $\mathrm{CO}_{2}$ between times 0.2 and 1 Sec., and three indices used the second derivative of the capnographic curve $\left(\mathrm{SD}_{1}\right.$, $\mathrm{SD}_{2}$, and $\mathrm{SD}_{3}$ ). Their results show large differences among the indices. According to their research, a high sensitivity to airway obstruction was seen for intermediate and terminal slopes $\left(S_{2}, S_{3}, S R\right)$, followed by $\mathrm{SD}_{1}, \mathrm{SD}_{2}, \mathrm{SD}_{3}$, and the lowest ranges were seen with $\mathrm{S}_{1}$ and $\mathrm{AR}$. This research introduces some basic and useful parameters. But it is a manual analysis of capnogram and lead to mistake due to human factors such as lack of expertise.

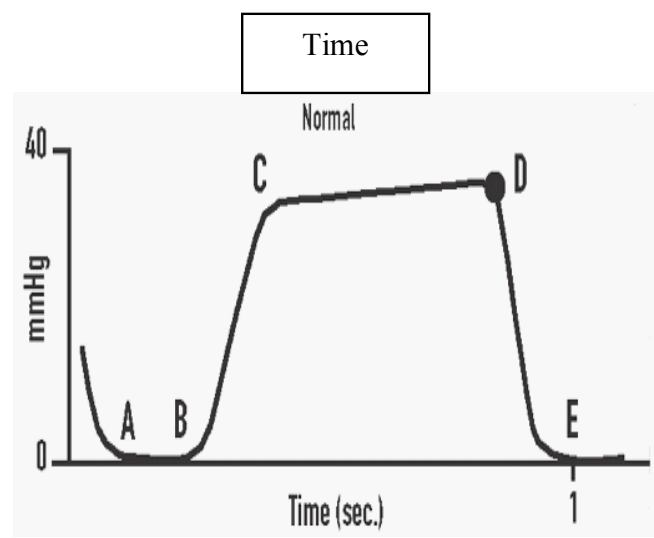

(a)

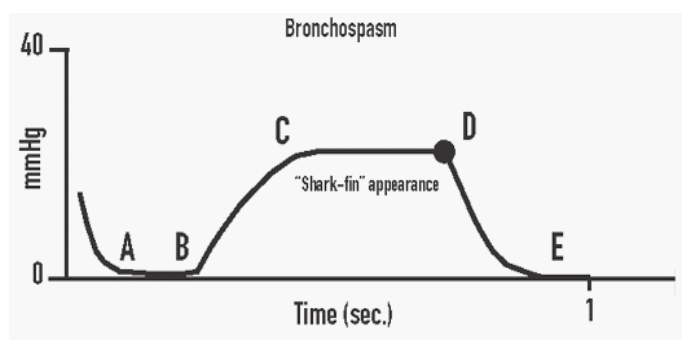

(b)

Figure 2: Comparing Waveforms, (a) Normal and (b) Bronchospastic
In 1996, Michael Yaron et al. [8] conducted a research on utility of the expiratory capnogram in the assessment of bronchospasm [8]. They calculated the $\mathrm{d}\left(\mathrm{CO}_{2}\right) / \mathrm{dt}$ of the plateau phase for five consecutive regular expirations. A mean calculated for each patient and conclude that this parameter is an effort-independent, rapid, non-invasive measured. It indicates significant bronchospasm in adult patients with asthma. Their study was on just 20 adults with acute asthma and 28normal adults. Like previous study, this research defines a new parameter to analyse the capnogram for asthmatic patients, but it is manual and difficult to calculate by a medical practitioners and physicians to monitor severity of asthma when they are monitoring the patients. Another research is by Druck, et al. [9]. They have evaluated the slope of phase III from the volumetric capnogram as a non-effort dependent surrogate for changes in peak expiratory flow rate. The patients breathed quietly for at least one minute through a combined $\mathrm{CO}_{2}$ /flow sensor and the best of three peaks expiratory flow rate measurements were then recorded. After that average values of the slope of phase III were computed over a 10-breath interval. Then percentage changes in slope measurements from the volumetric capnogram were compared to percentage changes in peak expiratory flow rates. The patient's correlation coefficient was calculated. This study has just suggested that changes in the volumetric capnogram slope of phase III may be useful as a non-effort dependent surrogate of peak expiratory flow rate and a measure of bronchospasm. However, it also needs lots of manual computing.

As mentioned above, all previous researches show significant correlation between the capnogram and asthmatic patient. However all of them are just manual study conducted through the conventional time domain method. In addition, manual analysis of capnogram is time-consuming and led to erroneous due to human factor such as tiredness and lack of expertise.

In this paper, an automated analysis of capnogram based on its shape is presented. The Linear Predictive Coding (LPC) coefficients are used for this purpose because these parameters are suitable for capnogram which has slope changes for asthmatic conditions [6].

The importance of linear prediction stems is from the fact that a signal wave and spectrum characteristics can be efficiently represented using a very small number of parameters. It means that, by minimizing the sum of the squared differences, over a finite interval, between the actual signal samples and the linearly predicted ones, a unique set of predictor coefficients can be determined that are the weighting coefficients used in the linear combination $[10,11]$. The ideas of linear prediction have been in use in the areas of control, and information theory under the names of system estimation and system identification [12].

\section{Methods}

In this section, 3 steps are presented which start with data Collection. Then LPC coefficients extraction method is discussed. Lastly, the effectiveness of the extracted coefficients is validated.

\section{Data acquisition}

The capnogram data were collected from patients in Penang Hospital Emergency Department for complaint about asthma and breathing difficulties. First, the patients were attached with capnography sensor on mouth or nose. Mainstream capnography method is suggested and used in the data collection because mainstream method shows higher accuracy [13].

After attaching the sensor on the patient's nose or mouth, the 
continuous capnogram was recorded using the capnography patient monitor, Nihon Kohden Bedside Monitor BSM-2301K. The data and the capnogram in the patient monitor were then extracted to personal computer for analysis. Throughout the study, 34 data were successfully collected with 18 non-asthmatic and 16 asthmatic patients capnogram recorded with the length of 5 seconds and frequency sampling of 1 $\mathrm{KHz}$.

\section{LPC Method}

Firstly the discrete signal sampled at every $\Delta \mathrm{T}$ (s) by $\left\{x_{t}\right\}$ is expressed. The frequency range of signal is $0-\mathrm{W}(\mathrm{Hz})$ and $\Delta \mathrm{T}$ must satisfy $\Delta \mathrm{T}$ $\leq 1 / 2 \mathrm{~W}$ (s). Assume the following first-order linear combination between the present sample value $x_{t}$ and the previous $p$ samples,

$$
x_{t}+\alpha_{1} x_{t-1}+\cdots+\alpha_{p} x_{t-p}=E_{t}
$$

Where $\{E\}$ is an uncorrelated statistical variable having a mean value of 0 and a variance of $\sigma^{2}$. This linear difference equation means that the present sample value $x_{t}$ can be linearly predicted using the previous sample values. The LPC algorithm developed to obtain the coefficients $\{a \downarrow i\}$ is as follow,

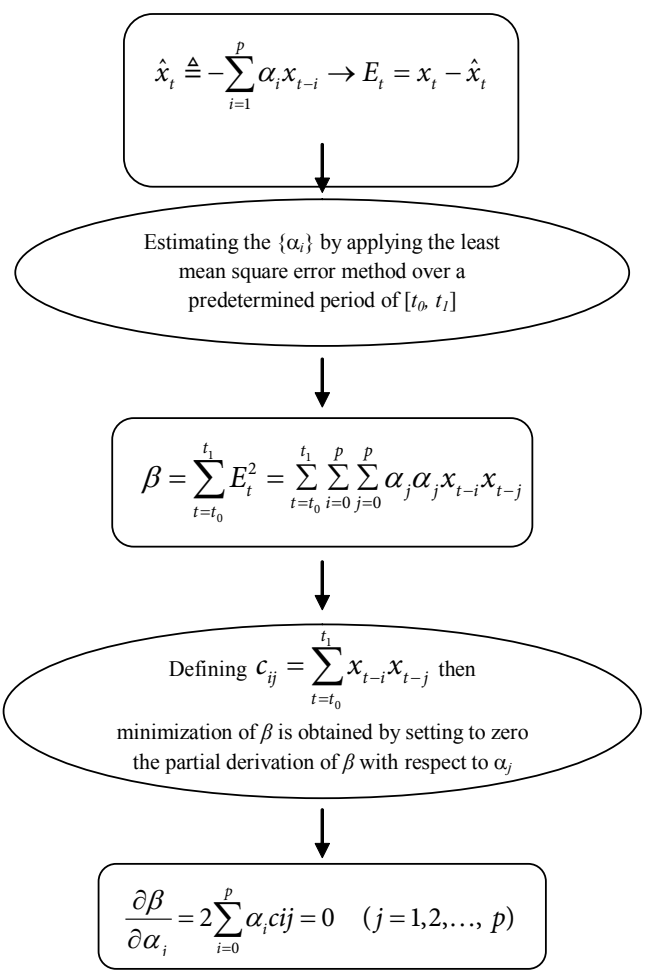

The autocorrelation method is used to solve the equation $\left(c_{i j}=\sum_{t=t_{0}}^{t_{1}} x_{t-i} x_{t-j}\right)$ by setting $t_{0}=-\infty$ and $t_{1}=\infty$, and by letting $x_{t}$ $=0$ for $\mathrm{t}<0$ and $\mathrm{t} \geq N[14,15,16]$. It means that, these limits allow $c_{i j}$ to be simplified as

$$
c i j=\sum_{t=-\infty}^{\infty} x_{t-i} x_{t-j}=\sum_{t=0}^{N-1-|i-j|} x_{t} x_{t+|i-j|}=r_{|i-j|}
$$

Thus, $\alpha_{i}$ is obtained by solving

$$
\sum_{t=0}^{p} \alpha_{i} r_{|i-j|}=0 \quad(j=1,2, \ldots, p)
$$

Although the error $E_{t}$ is minimized over an infinite interval, equivalent results are obtained by minimizing it only over $[0, N-1]$.

\section{Performance Measure}

The effectiveness of extracted coefficients is assessed by Receiver Operating Characteristic (ROC) curve analysis. ROC curves can be used to compare the diagnostic performance of two or diagnostic tests [17]. It considers the results of a particular test in two categories; one population with a disease, and the other population without the disease. It will rarely observe a perfect separation between the two groups.

When the variable under study cannot distinguish between the two groups, i.e. where there is no difference between the two distributions, the area under the ROC curve will be equal to 0.5 , so the ROC curve will coincide with the diagonal. When there is a perfect separation of the values of the two groups, i.e. there is no overlapping of the distributions, the area under the ROC curve equals 1 , so the ROC curve will reach the upper left corner of the plot. Also the P-value is the probability that the sample Area under the ROC curve is found when the true population area under the ROC curve is 0.5 (null hypothesis: Area $=0.5$ ). If $\mathrm{P}$ is low $(\mathrm{P}<0.05)$ then it can be concluded that the area under the ROC curve is significantly different from 0.5 and that therefore there is evidence that the capnogram test does have an ability to distinguish between the two groups [18].

\section{Results and Discussion}

In this section, firstly, the result for LPC order determination is presented. LPC coefficients are shown. After that, the performance of LPC coefficients to differentiate the asthmatic patients from the nonasthmatic patients is evaluated.

The matter of fact is that, the number of predictor parameters, $p$, depends primarily on the frequency range and is essentially independent of the LPC method being used [10]. So, for this analysis, we use LPC with order $8(p=8)$ because capnogram is related to breathe. The average respiratory rate reported in a healthy adult at rest is usually given as 12 breaths per minute, but estimates do vary between sources, although according to be healthy, unhealthy, and age it could change. However totally it is between 12 to 50 breaths per minute [19], therefore, it has low frequency range, and using LPC with order

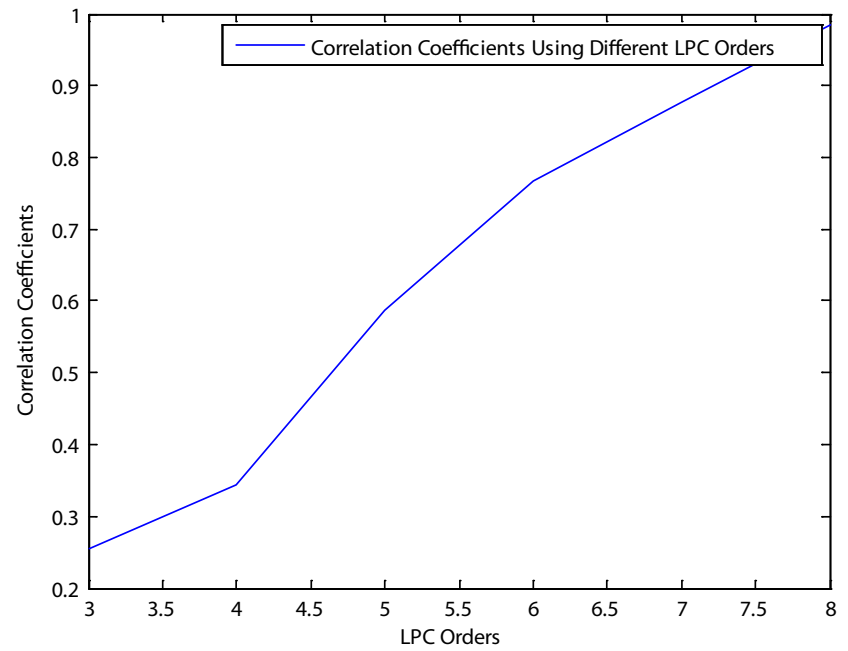

Figure 3: Correlation coefficients between the original capnogram signal and estimated signals using different LPC orders. 


\begin{tabular}{|l|l|l|l|l|l|l|l|}
\hline Samples & $\boldsymbol{\alpha}_{\mathbf{1}}$ & $\boldsymbol{\alpha}_{\mathbf{2}}$ & $\boldsymbol{\alpha}_{\mathbf{3}}$ & $\boldsymbol{\alpha}_{\mathbf{4}}$ & $\boldsymbol{\alpha}_{\mathbf{5}}$ & $\boldsymbol{\alpha}_{\mathbf{6}}$ & $\boldsymbol{\alpha}_{\mathbf{8}}$ \\
\hline CAPs & -1.26144 & 0.094706 & 0.081875 & 0.0129937 & -0.00660 & 0.0180562 & -0.01433 \\
\hline CNPs & -1.33359 & 0.224122 & -0.12026 & 0.106344 & -0.00047 & 0.0719833 \\
\hline
\end{tabular}

Table 1: The mean of LPC coefficients for CAP and CNP samples.

\begin{tabular}{|l|l|l|}
\hline LPC Coefficients & AUC & P-Value \\
\hline $\boldsymbol{\alpha}_{1}$ & 0.649 & 0.1384 \\
\hline $\boldsymbol{\alpha}_{2}$ & 0.625 & 0.2096 \\
\hline $\boldsymbol{\alpha}_{3}$ & 0.727 & 0.0096 \\
\hline $\boldsymbol{\alpha}_{4}$ & 0.705 & 0.0281 \\
\hline $\boldsymbol{\alpha}_{5}$ & 0.691 & 0.0507 \\
\hline $\boldsymbol{\alpha}_{6}$ & 0.712 & 0.0232 \\
\hline $\boldsymbol{\alpha}_{\mathbf{1}}$ & 0.625 & 0.2313 \\
\hline $\boldsymbol{\alpha}_{8}$ & 0.559 & 0.5669 \\
\hline
\end{tabular}

Table 2: The AUC and P-Value for results.

8 is suitable. Figure 3 shows the correlation coefficients between the original signal and estimated signals using different LPC orders.

Table 1 shows the mean of LPC coefficients for both CAP and CNP samples. From the table it can be seen that the mean of $\alpha_{2}, \alpha_{3}, \alpha_{4}, \alpha_{5}$, and $\alpha_{6}$ have significant difference between asthmatic and non-asthmatic patients. However, it needs to be approved by ROC method.

Table 2 shows the AUC and P-Value for LPC coefficients. In general, all coefficients has AUC $>0.5$ and $\mathrm{p}$ value $<0.2$. This shows that almost all coefficients are able to differentiate the asthmatic condition and the non-asthmatic condition. However, compared to all the coefficients, it can be seen from table 2 that, $\alpha_{3}, \alpha_{4}, \alpha_{5}$, and $\alpha_{6}$ have significant AUC and p-value which is efficient to make the capnogram data in two groups. This shows these LPC coefficients can significantly differentiate the asthmatic conditions.

Therefore as it shown in Table $2, \alpha_{3}, \alpha_{4}, \alpha_{5}$, and $\alpha_{6}$ are the best among LPC coefficients of samples to differentiate asthmatic conditions.

\section{Conclusion}

Current methods of monitoring asthma possess limitations. Therefore, monitoring asthma by using capnography is an alternative way to overcome the limitations. However, manual analysis of capnogram is time-consuming and led to erroneous due to human factor such as tiredness and lack of expertise. In this paper, an automated way to differentiate asthmatic conditions is presented. The results show that by using LPC analysis of capnogram and extracting coefficients $\alpha_{3}, \alpha_{4}, \alpha_{5}$, and $\alpha_{6}$, the asthmatic conditions can be differentiate with good accuracy, also the result can be used to design an artificial neural network to enhance the proposed method. This method is an innovative idea that is useful for healthcare professional involved in respiratory care as it would be possible to monitor severity of asthma automatically.

\section{References}

1. Guyton AC, Hall JE (2006) Medical Physiology $11^{\text {th }}$ edition Elsevier Inc.

2. Christopher HF, Elisabeth SS, Elaine LC, Kenan EH (2007) The Asthma Educator's Handbook McGraw-Hill.
3. James TL (2006) Pharmacotherapy of Asthma Taylor \& Francis Group.

4. Rhoades C, Thomas F (2002) Capnography: Beyond the Numbers. Air Med J 21: 43-48.

5. Grady SG, Egstrom S, Fisher JA (1993) Continuous end-tidal carbon dioxide monitoring. AACN proce manu crit care 206-212.

6. Egleston CV, Ben Aslam H, Lambert MA (1997) Capnography for monitoring non-intubated spontaneously breathing patients in an emergency room setting. J Accid Emerg Med 14: 222-224.

7. You B, Peslin R, Duvivier C, Vu VD, Grilliat JP (1994) Expiratory capnography in asthma: evaluation of various shape indices. Eur Respir J 7: 318-323.

8. Yaron M, Padyk P, Hutsinpiller M, Cairns CB (1996) Utility of the Expiratory Capnogram in the Assessment of Bronchospas Ann of Emerg Med 28: 403407

9. Druck J, Rubio PM, Valley MA, Jaffe MB, Yaron M (2007) Evaluation of the Slope of Phase III from the Volumetric Capnogram as a Non-Effort Dependent in Acute Asthma Exacerbation. An Emerg Med 50: 130-131.

10. Rabiner LR, Schafer RW (1978) Digital Processing of Speech Signals PrenticeHall.

11. Furui S (2001) Digital Speech Processing, Synthesis, and Recognition Second Edition, Marcel Dekker.

12. Deller JR, Hansen JH, Proakis JG (2000) Discrete-time processing of Speech Signals John Wiley and Sons Inc.

13. Swenson J, Henao-Guerrero PN, James WC (2008) Clinical Technique: Use of Capnography in Small Mammal Anesthesia. J Exo Pet Med 17: 175-180.

14. Zhao LV, Xiaopei Wu, Mi Li (2009) A research on EOG Feature Parameters Extraction Based on Linear Predictive Coding Model. Intern Conf Bioinf \& Biomed Eng 1-4.

15. Guilin Ma, Gran F, Jacobsen F, Agerkvist T (2011) Adaptive Feedback Cancellation with Band-Limited LPC Vocoder in Digital Hearing Aids IEEE Transaction on Audio, Speech, and Language Processing 19: 677-687.

16. Han H, Cho S, Chong U (2010) Fault Diagnosis System using LPC Coefficients and Neural Network. Inter Conf Signal Proce Sys 87-90.

17. Zweig MH, Campbell G (1993) Receiver-operating characteristic (ROC) plots: a fundamental evaluation tool in clinical medicine. Clin Chem 39: 561-577.

18. Griner PF, Mayewski RJ, Mushlin Al, Greenland P (1981) Selection and Interpretation of Diagnostic Tests and Procedures. Principles and applications. Ann Intern Med 94: 557-592.

19. Sherwood L (2006) Fundamentals of Physiology: A Human Perspective. Thomson Brooks and Cole Inc. 